\title{
A method for resummation of perturbative expansions based on the stochastic solution of Schwinger-Dyson equations
}

\author{
Pavel Buividovich* ${ }^{* \dagger}$ \\ ITEP, Moscow and JINR, Dubna \\ E-mail: buividovich@itep.ru
}

\begin{abstract}
We describe a numerical method for resummation of perturbative expansions in quantum field theories, which is based on the stochastic solution of Schwinger-Dyson equations. Our algorithm randomly generates open Feynman diagrams with probability proportional to their weight times some factor which compensates the combinatorial growth of their number. Perturbative series can be then easily re-summed by a Pade-Borel-Leroy procedure. As a simple test of our method, we apply it to a theory of one-component scalar field with quartic interaction. Resummation of perturbative expansion of renormalized coupling constant confirms that this theory is trivial in four and five space-time dimensions, and that the trivial fixed point is unstable in three dimensions.
\end{abstract}

XXIX International Symposium on Lattice Field Theory

July 10 - 162011

Squaw Valley, Lake Tahoe, California

\footnotetext{
* Speaker.

${ }^{\dagger}$ This work was partly supported by Grants RFBR 09-02-00338-a, RFBR 11-02-01227-a, NSh-6260.2010.2 and by the FAIR-Russia Research Center (FRRC).
} 
Monte-Carlo integration over all possible field configurations is one of the most universal and popular numerical methods for simulations of quantum field theories. However, such method might experience significant slow-down when a typical correlation length of quantum fields becomes very large. As well, it cannot be applied if the path integral weight is non-positive, which is often the case for fermionic field theories.

An alternative to the standard Monte-Carlo is the so-called Diagrammatic Monte-Carlo, which randomly samples weak- or strong-coupling expansion diagrams rather than field configurations. In many cases, Diagrammatic Monte-Carlo significantly reduces critical slowing-down and the severity of the sign problem $[1,2]$. For this reason, it is also believed that such diagrammatic methods might help to solve a famous problem of simulating QCD at finite chemical potential.

A serious obstacle for Diagrammatic Monte-Carlo is the factorial divergence of the weakcoupling perturbative expansions. Namely, the number of Feynman diagrams grows factorially with the diagram order, and the resulting series cannot be directly summed. For this reason, Diagrammatic Monte-Carlo was applied mostly to strong-coupling expansions, which can be analytically continued to the weak-coupling regime in a finite volume [2]. Unfortunately, strong-coupling expansions are often quite complicated, and it is not always clear how to fit them into Diagrammatic Monte-Carlo. For instance, it is not known how to sample strong-coupling expansion diagrams in $S U(N)$ gauge theories or $S U(N)$ sigma-models. Moreover, for such theories the continuum limit is approached in the weak-coupling regime, and one can expect numerical methods based on the weak-coupling expansion to be more efficient. A method for approximation of the sum of divergent series by a sequence of functions with convergent expansions has been proposed recently in [3], however, so far it has been applied to zero-dimensional path integrals only. It is also not clear how to generalize the approach of [3] to lattice field theories with compact variables, such as lattice QCD. Thus, up to now a systematic method which would be able to perform a Monte-Carlo (re)summation of conventional Feynman diagrams is not known.

In this Letter we describe a simulation strategy which can be applied to theories with asymptotic perturbative expansions and which is formulated without any explicit reference to diagram weights. Much like the "worm" algorithm [1], our method generates open Feynman diagrams by some random process. This process is constructed in such a way that its stationary probability distribution satisfies the perturbatively expanded Schwinger-Dyson equations of the theory. Since Schwinger-Dyson equations can be easily derived for any quantum field theory, we believe that our method has a large potential for generalizations. In [4] we have already applied a similar idea to quantum field theories in the large- $N$ limit, where saddle points of path integrals correspond to sums over planar diagrams. Here we extend our approach beyond the planar approximation.

In order to illustrate our method, we consider Schwinger-Dyson equations for the theory of a single-component scalar field with quartic interaction. The action of the theory is:

$$
S=\int d^{D} x\left(1 / 2 \partial_{\mu} \phi \partial_{\mu} \phi+m_{0}^{2} / 2 \phi^{2}+\lambda_{0} / 4 \phi^{4}\right)
$$

We now define the disconnected field correlators in momentum space as $G\left(p_{1}, \ldots, p_{n}\right)=$ $\left\langle\phi\left(p_{1}\right) \ldots \phi\left(p_{n}\right)\right\rangle, \phi(p)=\int d^{D} x e^{i p x} \phi(x)$. We also omit the trivial factor $(2 \pi)^{D} \delta\left(\sum_{A=1}^{n} p_{A}\right)$ from all the correlators and assume that the momenta entering them are conserved. 
Schwinger-Dyson equations for the theory (1), obtained by varying the path integral over $\phi\left(p_{1}\right)$, are:

$$
\begin{array}{r}
\left(m^{2}+p_{1}^{2}\right) G\left(p_{1}, p_{2}, \ldots, p_{n}\right)=\sum_{A=2}^{n}(2 \pi)^{D} \delta\left(p_{1}+p_{A}\right) G\left(p_{2}, \ldots, p_{A-1}, p_{A+1}, \ldots, p_{n}\right)- \\
-\frac{\lambda_{0}}{(2 \pi)^{2 D}} \int d^{D} q_{1} d^{D} q_{2} d^{D} q_{3} \delta\left(p_{1}-q_{1}-q_{2}-q_{3}\right) G\left(q_{1}, q_{2}, q_{3}, p_{2}, \ldots, p_{n}\right),
\end{array}
$$

where the arguments of the correlator in the first summand on the r.h.s. of (2) are all the momenta except $p_{1}$ and $p_{A}$. We thus arrive at a complete system of functional linear equations for an infinite set of unknown functions $G\left(p_{1}, \ldots, p_{n}\right)$.

Infinite systems of equations similar to (2) can be efficiently solved by stochastic methods (see e.g. [5] for a review). Following our earlier work [4], we would like to interpret the equations (2) as equations for the stationary probability distribution of some Markov process. Such equations have the general form $\omega(A)=\sum_{B} P(B \rightarrow A) \omega(B)$, where $\omega(A)$ is the stationary probability to encounter the state $A$ and $P(B \rightarrow A)$ is the probability of transition from $B$ to $A$. The space of states of the required process should contain the sequences of momenta $\left\{p_{1}, \ldots, p_{n}\right\}$, and the correlator $G\left(p_{1}, \ldots, p_{n}\right)$ should be proportional to the probability of encountering the sequence $\left\{p_{1}, \ldots, p_{n}\right\}$. Since the random process would not be able to reach the configurations of momenta $\left\{p_{1}, \ldots, p_{n}\right\}$ with sufficiently small $G\left(p_{1}, \ldots, p_{n}\right)$ in a finite time, an infinite hierarchy of equations (2) would be automatically truncated.

It turns out, however, that a direct stochastic interpretation of equations (2) is impossible. The reason is that the probability to go from the state with $n$ momenta to the state with $n+2$ momenta, which is proportional to the number of summands in the first term on the r.h.s. of (2), for sufficiently large $n$ always exceeds unity. This is, in fact, already the manifestation of the factorial divergence of the perturbative series. Note the difference with large- $N$ quantum field theories, where perturbative series converge at sufficiently small coupling and a direct stochastic interpretation of SchwingerDyson equations is possible [4].

Here we propose to cast the equations (2) into stochastic form by formally expanding the field correlators in powers of the bare coupling constant $\lambda_{0}$ :

$$
G\left(p_{1}, \ldots, p_{n}\right)=\sum_{m=0}^{+\infty} c_{n, m}\left(-\lambda_{0}\right)^{m} G_{m}\left(p_{1}, \ldots, p_{n}\right),
$$

with some coefficients $c_{n, m}$. Schwinger-Dyson equations (2) then relate expansion coefficients with different $m$ :

$$
\begin{array}{r}
G_{m}\left(p_{1}, \ldots, p_{n}\right)=\sum_{A=2}^{n} \frac{c_{n-2, m}(2 \pi)^{D} \delta\left(p_{1}+p_{A}\right)}{c_{n, m}\left(m_{0}^{2}+p_{1}^{2}\right)} G_{m}\left(p_{2}, \ldots, p_{A-1}, p_{A+1}, \ldots, p_{n}\right)+ \\
+\frac{c_{n+2, m-1}}{c_{n, m}(2 \pi)^{2 D}\left(m_{0}^{2}+p_{1}^{2}\right)} \int d^{D} q_{1} d^{D} q_{2} d^{D} q_{3} \delta\left(p_{1}-q_{1}-q_{2}-q_{3}\right) G_{m-1}\left(q_{1}, q_{2}, q_{3}, p_{2}, \ldots, p_{n}\right),
\end{array}
$$

where we assume that $G_{m}$ with no arguments is proportional to $\delta_{m, 0}$ and $c_{n, m}=0$ for $m<0$.

In order to compensate for the growth of the number of summands in the first term on the r.h.s. of (4), the coefficients $c_{n, m}$ should grow not slower than $(n / 2)$ ! at large $n$. The contribution of the 
second term remains finite for any $n$ and $m$ if $c_{n, m}$ grow as $(n / 2+m)$ ! at large $n, m$. After some optimization we arrive at the following choice of the coefficients $c_{n, m}$ :

$$
c_{n, m}=\Gamma(n / 2+m+1 / 2) x^{-(n-2)} y^{-m},
$$

where $x^{2}=\frac{1}{4(2 \pi)^{D} \Sigma_{0}}, y=\frac{(2 \pi)^{D} m_{0}^{2}}{8 \Sigma_{0}}, \Sigma_{0}=\int_{|q|<\Lambda} d^{D} q \frac{1}{q^{2}+m_{0}^{2}}$ and $\Lambda$ is the ultraviolet cutoff.

With $c_{n, m}$ given by (5), we can interpret equations (4) as equations for the stationary probability distribution of a Markov process with states specified by sequences of momenta $\left\{p_{1}, \ldots, p_{n}\right\}$ and nonnegative integer numbers $m$. It is also convenient to extend this space of states by adding a real number $\chi \in[0,1]$. We describe this random process as

Algorithm 1. At each iteration, do one of the following:

Add momenta: With probability $p_{A}=\frac{n+1}{2 n+4 m+2}$ insert a random momentum $p$ at the beginning of the sequence $\left\{p_{1}, \ldots, p_{n}\right\}$, and $-p$ - between the $A$ 'th and $A+1$ 'th elements. $A$ is random in the range $0 \ldots n$. The probability distribution of $p$ is proportional to $\frac{1}{p^{2}+m_{0}^{2}}$, with $|p|<\Lambda . m$ and $\chi$ are not changed.

Create vertex: With probability $p_{V}=1 / 2$ remove the three first momenta from the sequence and replace them by their sum $p=p_{1}+p_{2}+p_{3}$. Multiply $\chi$ by $m_{0}^{2} /\left(m_{0}^{2}+p^{2}\right)$ and increase $m$ by one.

Restart: Otherwise set $\chi=1$ and $m=0$ and start with an empty sequence.

The solution of (4) is then given by $G_{m}\left(p_{1}, \ldots, p_{n}\right)=\mathscr{N} \int d \chi \chi \omega\left(\chi ; m ; p_{1}, \ldots, p_{n}\right)$, where $\omega\left(\chi ; m ; p_{1}, \ldots, p_{n}\right)$ is the probability to encounter the corresponding state of the random process and $\mathscr{N}$ is some normalization factor which can be found by comparing the probability of occurence of an empty sequence with unity. In practice, in order to measure $G_{m}\left(p_{1}, \ldots, p_{n}\right)$, one should weight each configuration of momenta by the factor $\chi$ when performing statistical averaging. $\mathrm{C}$ code of Algorithm 1 can be found at [6].

Feynman diagrams of the theory can be easily reconstructed from the history of our random process, hence the names of the actions in Algorithm 1. In particular, one can use only connected diagrams for measurements, which significantly reduces numerical noise. Factorial growth of the number of diagrams is compensated by the growth of coefficients $c_{n, m}$ in (3). The weight of each diagram is proportional to the kinematical factor

$$
\int \prod_{i} \frac{d^{D} q_{i}}{q_{i}^{2}+m_{0}^{2}} \prod_{j} \frac{1}{Q_{j}^{2}+m_{0}^{2}},
$$

where $q_{i}$ are independent momenta circulating in loops and $Q_{j}$ can be expressed as some linear combinations of $q_{i}$ and the momenta of the external legs. Our algorithm simply performs MonteCarlo integration over the independent momenta $q_{i}$, which are randomly distributed with probability $\sim\left(q_{i}^{2}+m_{0}^{2}\right)^{-1}$ within the $D$-dimensional spheres of radius $\Lambda$. The factor $\chi$ then contains the product of propagators involving $Q_{j}$.

Note also that transition probabilities for the Markov process specified by Algorithm 1 and hence its autocorrelation time (which does not exceed several iterations) do not depend on the 


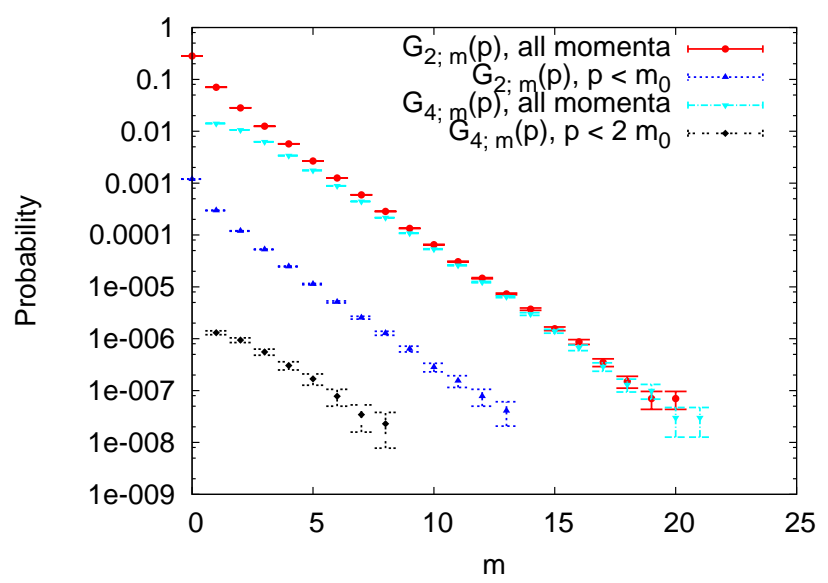

Figure 1: Probabilities of encountering connected diagrams with $m$ vertices and 2 and 4 external legs for Algorithm 1 in $D=4$, for any momenta on the external legs and for all momenta below some infrared cutoff. Solid lines illustrate the expected asymptotic behavior $\omega\left(\chi ; m ; p_{1}, \ldots, p_{m}\right) \sim p_{V}^{m}$ at large $m$.

physical parameters of the theory (1). Therefore Algorithm 1 does not slow down near the continuum limit in the sense of the standard Monte-Carlo. Analogue of this slowing down in our case is the growth of the number of iterations which are required to obtain sufficient statistics in the lowmomentum region. If $\Lambda_{I R}$ is the infrared cutoff, the volume of this region and hence the probability for all momenta to be within it decrease as $\Lambda_{I R}^{(n-1) D}$ as $\Lambda_{I R} \rightarrow 0$. Such kinematical suppression can be clearly seen on Fig. 1.

As a test of our algorithm, we have studied the renormalization-group trajectories for the theory (1) in different space-time dimensions, namely, the dependence of the renormalized coupling constant on the renormalized mass at fixed bare coupling constant $\lambda_{0}$. Renormalized mass $m_{R}$ is extracted from the two-point correlator at small momenta: $G(p)=\frac{Z_{R}}{m_{R}^{2}+p^{2}+O\left(p^{2}\right)}$, where $Z_{R}$ is the wave function renormalization constant. In practice we have considered the function $\Gamma_{2}(p)=\left(m_{R}^{2}+p^{2}\right) G(p)$, and tuned the value of $m_{R}$ so that $\Gamma_{2}(p)$ has minimal deviation from constant value $Z_{R}$ for $p<m_{R}$. Renormalized coupling $\lambda_{R}$ is related to the connected truncated fourpoint correlator $\Gamma\left(p_{1}, p_{2}, p_{3}, p_{4}\right)$ at zero external momenta: $\lambda_{R}=-1 / 6 Z_{R}^{2} \Gamma(0,0,0,0)$. We assess the zero-momentum limit of field correlators (or their expansion coefficients (3)) by summing up sequences of momenta generated by Algorithm 1 with an additional weight factor proportional to $\Lambda_{I R}^{-(n-1) D} \exp \left(-\sum_{A=1}^{n} \frac{p_{A}^{2}}{2 \Lambda_{I R}^{2}}\right)$. The result is then normalized so as to recover $G_{m}\left(p_{1}, \ldots, p_{n}\right)$ at $p_{1}, \ldots, p_{n}=0$ in the limit $\Lambda_{I R} \rightarrow 0$.

Once the expansion coefficients $G_{m}\left(p_{1}, \ldots, p_{n}\right)$ and the factors $c_{n, m}$ are known, the series (3) can be resummed using the Pade-Borel-Leroy procedure. Namely, in order to recover the correlators $G\left(p_{1}, \ldots, p_{n}\right)$, one should integrate their Borel-Leroy transform $G\left(z ; p_{1}, \ldots, p_{n}\right)=$ $\sum_{m=0}^{+\infty}(-z)^{m} G_{m}\left(p_{1}, \ldots, p_{n}\right)$ over $z$ with the weight proportional to $e^{-\frac{y z}{\lambda_{0}}} z^{\frac{n-1}{2}}$. Such integration reproduces the factors (5) in (3). Given only a finite number of known coefficients $G_{m}\left(p_{1}, \ldots, p_{n}\right)$, one approximates $G\left(z ; p_{1}, \ldots, p_{n}\right)$ with a rational function of $z$. This is equivalent to approximating the 


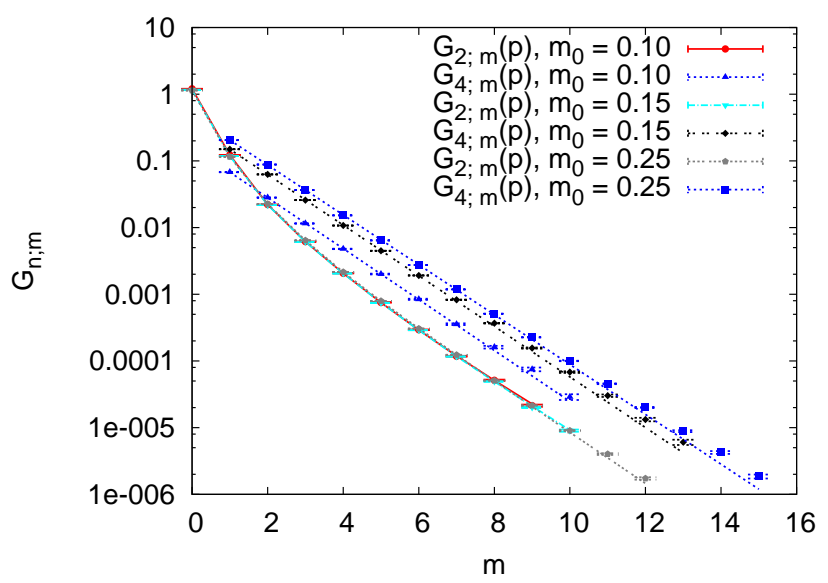

Figure 2: Reweighted expansion coefficients (3) for the regularized zero-momentum limit of two- and fourpoint connected truncated correlators $\Gamma_{2}\left(\Lambda_{I R}=m_{0}\right)$ and $\Gamma_{4}\left(\Lambda_{I R}=2 m_{0}\right)$ for $D=4$. Solid lines are the fits of the data with the sum of several exponents (three for the two-point, one for the four-point correlators). Only coefficients with relative error below $10 \%$ were used for fitting and are shown on the plot.

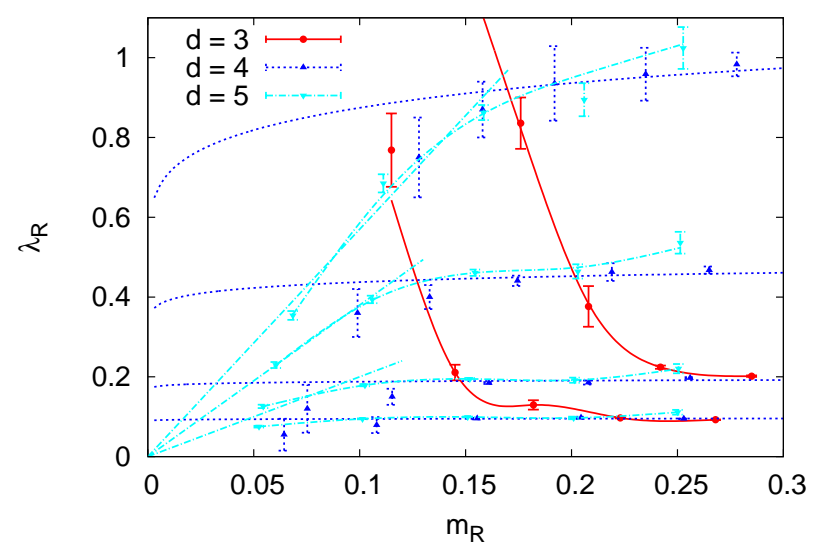

Figure 3: Renormalized coupling constant as a function of renormalized mass for fixed bare coupling $\lambda_{0}$. For $D=4$ and $D=5$ we take $\lambda_{0}=0.1,0.2,0.5,1.0$ and for $D=3, \lambda_{0}=0.1,0.2$. For $D=4$, solid lines correspond to the result of integration of one-loop massive $\beta$-function. For $D=5$, straight solid lines are the linear fits of the data at small masses. For $D=3$ and $D=5$, interpolating splines are also shown to guide the eye.

coefficients $G_{m}\left(p_{1}, \ldots, p_{n}\right)$ with a sum of exponential functions in $m: G_{m}\left(p_{1}, \ldots, p_{n}\right)=\sum_{k} a_{k} b_{k}^{m}$, where each summand corresponds to a simple pole of $G\left(z ; p_{1}, \ldots, p_{n}\right)$. Integration over $z$ can be then performed analytically. It turned out that standard Pade approximants constructed using all data points are very unstable due to statistical errors, and contain multiple spurious poles. In order to obtain stable approximants, we find the optimal number and values of $a_{k}$ and $b_{k}$ by fitting the numerical data. Such fits are illustrated on Fig. 2. A special fitting procedure based on the singular value decomposition of so-called Hankel matrices [7] was used. Our results are based on $10^{9}$ 
iterations of Algorithm 1 (taking several hours on a $2 \mathrm{GHz}$ CPU) for each $m_{0}$ and $D$.

The renormalized coupling constant $\lambda_{R}$ obtained with such a resummation procedure is plotted on Fig. 3 as a function of renormalized mass $m_{R}$ in lattice units. Renormalization-group arguments suggest that $\lambda_{R}$ should go to zero in the continuum limit $m_{R} \rightarrow 0$ for $D \geq 4$, and increase for $D<4$. Our results clearly agree with this "triviality" conjecture [2,8]. For $D=4$, the behavior of $\lambda_{R}$ is consistent within error range with the logarithmic scaling which follows from the integration of the one-loop $\beta$-function of the theory. As could be expected from dimensional arguments, for $D=5$ the dependence of $\lambda_{R}$ on $m_{R}$ is almost linear at small $m_{R}$. For $D=3 \lambda_{R}$ quickly grows as $m_{R}$ decreases. On the other hand, at large $m_{R}$ the renormalized coupling tends to its bare value $\lambda_{0}$ for all $D$.

We conclude that stochastic perturbative solution of Schwinger-Dyson equations, combined with a specially adopted Pade-Borel-Leroy resummation procedure, can be successfully used for numerical simulation of quantum field theories. It can be a viable alternative to Diagrammatic Monte-Carlo when weak-coupling perturbative expansions are only asymptotic.

\section{References}

[1] N. V. Prokof'ev, B. V. Svistunov, Phys.Rev.Lett. 81, 2514 (1998), ArXiv:cond-mat/9804097; ibid., Phys.Rev.Lett. 87, 160601 (2001).

[2] U. Wolff, Nucl.Phys.B 824, 254 (2010), ArXiv:0908.0284; ibid., Phys.Rev.D 79, 105002 (2009), ArXiv:0902.3100; ibid., Nucl.Phys.B 832, 520 (2010), ArXiv:1001.2231.

[3] L. Pollet, N. V. Prokof'ev and B. V. Svistunov, Phys.Rev.Lett. 105, 210601 (2010), ArXiv:1006.4519.

[4] P. V. Buividovich, Phys.Rev.D 83, 045021 (2011), ArXiv:1009.4033; ibid., ArXiv:1011.2664.

[5] A. Srinivasan, V. Aggarwal, Stochastic linear solvers, Proceedings of the SIAM Conference on Applied Linear Algebra, (2003).

[6] http://www.lattice.itep.ru/o7Epbaivid/codes.html

[7] P. De Groen, B. De Moor, J.Comput.Appl.Math. 20, 175 (1987).

[8] J. Fröhlich, Nucl.Phys.B 200, 281 (1982); K. G. Wilson, M. E. Fisher, Phys.Rev.Lett. 28, 240 (1972). 